\title{
La représentation du soi dans le monde littéraire et artistique : une instance mobile, plurielle et fragmentée
}

\author{
Laurence GAUVREAU, Université Simon Fraser \\ Hasheem HAKEEM, Université Simon Fraser
}

La manière de considérer la subjectivité, l'individu et l'instance du soi a souvent connu des changements majeurs, dont les plus récents influencent notre manière contemporaine de penser le soi. La révolution freudienne (Freud; Kristeva; Lacan) transforme d'abord notre conception de l'individu, puisque la notion d'inconscient, entre autres, remet en cause l'unité présumée des êtres humains. Le soi apparait ainsi discontinu, divisé et non entièrement maitrisé. Ensuite, l'avènement des idées postmodernes, peu importe si ce courant culturel et philosophique est considéré comme en rupture ou en continuité avec les idées modernes, suggère que tous les éléments de notre société sont socialement et culturellement construits, dont l'identité (Butler; Foucault ; Hutcheon ; Jameson ; Lyotard). Plutôt que d'être considéré comme une essence, le soi devient un concept bâti comme un amalgame non concordant d'influences culturelles et d'éléments identitaires qui proviennent de la société dans laquelle l'individu évolue. Puis, dans les années 1980, le postcolonialisme et plusieurs poussées migratoires laissent également voir que les structures cohérentes et l'identité stable tendent à être remplacées par le concept de culture et d'identité construites, relationnelles, en changement constant, avec des frontières poreuses, avec un sens toujours créé et renégocié. Finalement, en 1990, parait l'ouvrage influent de Paul Ricœur, Soi-même comme un autre, qui stipule que l'identité-ipse est une instance perçue contextuellement et subjectivement, fluctuant au fils du temps ou selon la perception changeante dont l'individu se saisit toujours « comme un autre ». Bref, découlant de ces nouvelles façons d'appréhender l'individu, les manières de représenter le soi semblent rencontrer des changements notables.

Le Pacte autobiographique établi par Philippe Lejeune en 1975 - « récit rétrospectif en prose qu'une personne réelle fait de sa propre existence, lorsqu'elle met l'accent sur sa vie individuelle, en particulier sur l'histoire de sa personnalité »(14) - se trouve ainsi remis en cause, ou du moins contredit par un nombre subséquent de récits de soi contemporains, puisque cette instance ne semble plus considérée par plusieurs comme concordante, fixe et maitre de son 
sens. Un nombre incalculable de théories du genre littéraire en découlent, tentant de saisir, catégoriser et décrire ce nouveau récit de soi : l'autofiction (Doubrovsky), la surfiction (Federman), les fictions de soi (Blanckeman), l'autonarration (Gasparini), les romans du je (Forest), etc.

Ce sont précisément ces diverses manières de représenter et d'écrire le soi qui nous ont amené.e.s à organiser un atelier intitulé : «Pour une cartographie des récits de soi : De l'autobiographie aux webcams » lors du congrès des sciences humaines à l'Université de la Colombie-Britannique en 2019, dans le but de réfléchir à cette question, entre autres, dans le domaine littéraire, cinématographique et artistique. Ce dossier, composé de cinq articles, traite ainsi du récit de soi aux origines multiples, en tant que cheminement ou parcours, comprenant l'Autre ou le récit familial, résistant aux identités figées et formé de plusieurs versions contradictoires de soi-même.

Dans le premier article, Adina Balint montre, à partir de La ballade d'Ali Baba de Catherine Mavrikakis, en dialogue avec L'énigme du retour de Dany Laferrière, comment le récit de la mobilité peut construire une identité narrative propre à l'espace et ainsi obliger la rencontre avec l'altérité. Quant à Miriem Méghaïzerou, elle propose une analyse du triptyque de Christophe Honoré : le roman Ton père (2017), le film Plaire, aimer et courir vite (2018) et la pièce de théâtre Les idoles (2018). Selon Méghaïzerou, le caractère multiple, insaisissable et fictionnel du «je » dans les œuvres d'Honoré permet à «l'artiste homosexuel » de se réinventer dans le but d'échapper à la catégorisation essentialiste, immuable et réductrice de l'identité. Dans son étude de l'usage de l'objet dans la pratique autobiographique de Collette Fellous, Tara Collington s'intéresse à la relation entre la mémoire et les accessoires du passé dans Avenue de France (2001) et Un amour de frère (2011). Selon Collington, les objets-mémoire (visibles et invisibles) de Fellous servent une écriture du soi fondée sur un certain accès au passé et au récit familial. Pour sa part, Megan Wightman étudie l'écriture autobiographique de Maurice Béjart en tant qu'artiste de la danse. L'auteure constate que les mémoires de Béjart ne s'avèrent pas une version fictionnalisée de son récit de vie, mais plutôt une performance littéraire de l'identité artistique plurielle de Béjart. Finalement, partant du principe postmoderne que le soi est socialement construit, Laurence Gauvreau se penche sur la question de l'intertextualité et fait ressortir la manière dont ce procédé permet de construire une identité littéraire. 
Pour conclure, nous aimerions remercier la directrice de rédaction de Voix plurielles, Catherine Parayre, d'avoir accepté de publier ce dossier. Nos reconnaissances vont également aux auteur.e.s des articles pour leur travail exemplaire et leur précieuse collaboration, ainsi qu'aux évaluateurs et évaluatrices, dont les commentaires à la fois constructifs et consciencieux ont sans doute contribué à améliorer la qualité de ce dossier.

\section{Bibliographie}

Blanckeman, Bruno. Les fictions singulières, étude sur le roman français contemporain. Paris : Prétexte, 2002.

Butler, Judith. Gender Trouble, Feminism and the Subversion of Identity. New York : Routledge, 1990.

Doubrovsky, Serge. Fils. Paris : Galilée, 1977.

Federman, Raymond. Surfiction. Marseille : Le Mot et le Reste, 2006.

Forest, Philippe. Le roman, le je. Nantes : Pleins Feux, 2001.

Foucault, Michel. «Le sujet et le pouvoir ». Dits et écrits - tome IV. Paris : Gallimard, 1994.

Freud, Sigmund. L'interprétation du rêve (Die Traumdeutung). Vienne : Deuticke, 1990.

Gasparini, Philippe. Est-il je ? Roman autobiographique et autofiction. Paris : Seuil, 2004.

Hutcheon, Linda. The Politics of Postmodernism. New York : Routledge, 1989.

Jameson, Fredric. «The Cultural Logic of Late Capitalism ». New Left Review 146 (1984). 5392.

Kristeva, Julia. Étrangers à nous-mêmes. Paris : Gallimard, 1988.

Lacan, Jacques. The Language of the Self: The Function of Language in Psychoanalysis. Baltimore : Johns Hopkins UP, 1998 (1968).

Lejeune, Philippe. Le pacte autobiographique. Paris : Seuil, 1975.

Lyotard, Jean-François. La condition postmoderne. Paris : Minuit, 1979.

Ricœur, Paul. Soi-même comme un autre. Paris : Seuil, 1990. 